\title{
Cladding-pumped Yb-doped fiber laser with vortex output beam
}

\author{
D. Lin, W. A. Clarkson \\ Optoelectronics Research Centre, University of Southampton, Southampton, SO17 1BJ, UK \\ di.lin@soton.ac.uk
}

\begin{abstract}
A simple technique for selectively generating a donut-shaped $\mathrm{LP}_{11}$ mode with vortex phase front in a cladding-pumped ytterbium-doped fiber laser is reported. The laser yielded $34 \mathrm{~W}$ of output with a slope efficiency of $74 \%$.

OCIS codes: (140.3510) Laser, fiber; (260.5430) Polarization; (080.4865) Optical vortices
\end{abstract}

\section{Introduction}

Recently, operation on individual higher-order modes in fiber lasers has attracted growing interest due to the potential for larger mode area, which, in turn, raises the threshold for damage and deleterious nonlinear loss processes allowing further scaling of output power and pulse energy. Further benefits in terms of applications potential can be derived from the unique properties (i.e. intensity profile, polarization and phase distribution) of some of these higher order modes. One notable example is the optical vortex beam characterized by a donut-shaped intensity distribution with a helical phase front that carries orbital angular momentum (OAM). Optical vortex beams have applications in a growing number of areas including, micro-particle manipulation, quantum information and laser processing of materials. The $\mathrm{LP}_{11}$ family modes comprise four vector modes; $\mathrm{TE}_{01}, \mathrm{HE}_{21}^{\text {even }}, \mathrm{HE}_{21}^{\text {odd }}$, and $\mathrm{TM}_{01}$, and it can be shown that the coherent superposition of two vector modes (i.e. $\mathrm{HE}_{21}^{\text {even }}$ and $\mathrm{HE}_{21}^{\text {odd }}, \mathrm{TE}_{01}$ and $\mathrm{TM}_{01}$ ) with $\pm \pi / 2$ phase difference between them will lead to circularly-polarized optical vortex beams carrying $\pm \hbar$ of OAM per photon [1]. In the scalar approximation, the optical vortex beams can be considered as a linear combination of two orthogonal two-lobed $\mathrm{LP}_{11}$ modes with $\pm \pi / 2$ phase shift between them, which was demonstrated in a passive multi-mode fiber [2] and an Yb-doped fiber amplifier [3], where the phase shift was achieved by induced stress. In this paper, for brevity, we designate beams with a donut-shaped intensity distribution in the $\mathrm{LP}_{11}$ family modes or via superposition of these modes as the donut-shaped $\mathrm{LP}_{11}$ modes.

Here, we report a very simple strategy for achieving lasing on the first higher-order donut shaped $\mathrm{LP}_{11}$ mode (or the fundamental $\mathrm{LP}_{01}$ mode) in a few-moded $\mathrm{Yb}$-doped fiber laser with high efficiency and high mode quality using an intracavity polarization filter exploiting the difference in polarization behavior believed to be due to different effective birefringence and orientation of fast and slow axes for each mode $[4,5]$.

\section{Experiment and Results}

The laser configuration used in our experiments is shown in Fig. 1. A 1m length of non-polarization maintaining Yb-doped double-clad fiber was employed as the gain medium. The latter had a circular core of diameter, $20 \mu \mathrm{m}$ and numerical aperture (NA), 0.08, surrounded by an octagonal silica inner-cladding of dimension, $125 \mu \mathrm{m}$. The Vnumber for the core was calculated to be 4.72 at $1064 \mathrm{~nm}$ guaranteeing that only the fundamental $\mathrm{LP}_{01}$ and its neighboring $\mathrm{LP}_{11}$ mode could be guided within the fiber. Feedback for lasing was provided by the $\sim 3.6 \%$ Fresnel reflection from a perpendicularly-cleaved fiber facet at the laser output end of the fiber and by an external feedback cavity at the opposite end. The latter incorporated an aspheric collimating lens of focal length, $18.4 \mathrm{~mm}$, a high reflectivity plane mirror, a polarizing beam splitter and a zero-order half-wave $(\lambda / 2)$ plate at $1064 \mathrm{~nm}$ mounted on rotation stage. This combination ensured that a linearly-polarized beam was fed-back into the fiber, which could be aligned at any desired angle via simple adjustment of the half-wave plate. The end of the fiber adjacent to the external cavity was cleaved at $8^{\circ}$ to suppress the broadband feedback and hence parasitic lasing between the fiber

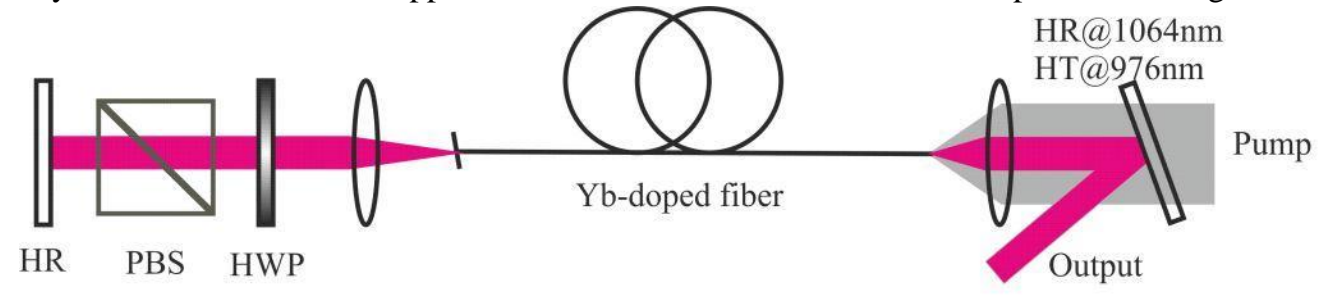

Fig. 1 Experimental set-up: High reflective mirror (HR), polarization beam splitter (PBS), and half-wave plate (HWP). 
end facets. Pump light was provided by a $60 \mathrm{~W}$ fiber-coupled $976 \mathrm{~nm}$ laser diode with a $105 \mu \mathrm{m}$ core diameter and $0.12 \mathrm{NA}$, which was free-space coupled into the output end of the fiber via a dichroic mirror.

It was found that the fundamental $\mathrm{LP}_{01}$ or donut-shaped $\mathrm{LP}_{11}$ modes could be selected at will by simply rotating the fast-axis of the $\lambda / 2$ waveplate to different angles. When the orientation of fiber was adjusted appropriately, the operation for the $\mathrm{LP}_{01}$ mode was achieved by setting the fast-axis of $\lambda / 2$ waveplate to be in horizontal direction (i.e. so that the polarization direction was parallel to the plane of Fig. 1), whereas the donut-shaped $\mathrm{LP}_{11}$ mode could be selected by adjusting the orientation of fast-axis of the $\lambda / 2$ waveplate to be $\sim 22.5^{\circ}$ (i.e. so that polarization direction for the beam coupled into the fiber was at $\sim 45^{\circ}$ to the plane of Fig.1). It was evident that both modes had a similar characteristic of output power versus pump power as illustrated in Fig. 2(a). Both modes reached threshold at a pump power of $\sim 1.4 \mathrm{~W}$ (absorbed) and their power increased linearly with pump power to an output power of $36 \mathrm{~W}$ at a lasing wavelength of $\sim 1040 \mathrm{~nm}$ at the maximum available pump power (52 W absorbed). The corresponding slope efficiency with respect to absorbed pump power was $\sim 74 \%$. Additionally, the output beams had approximately
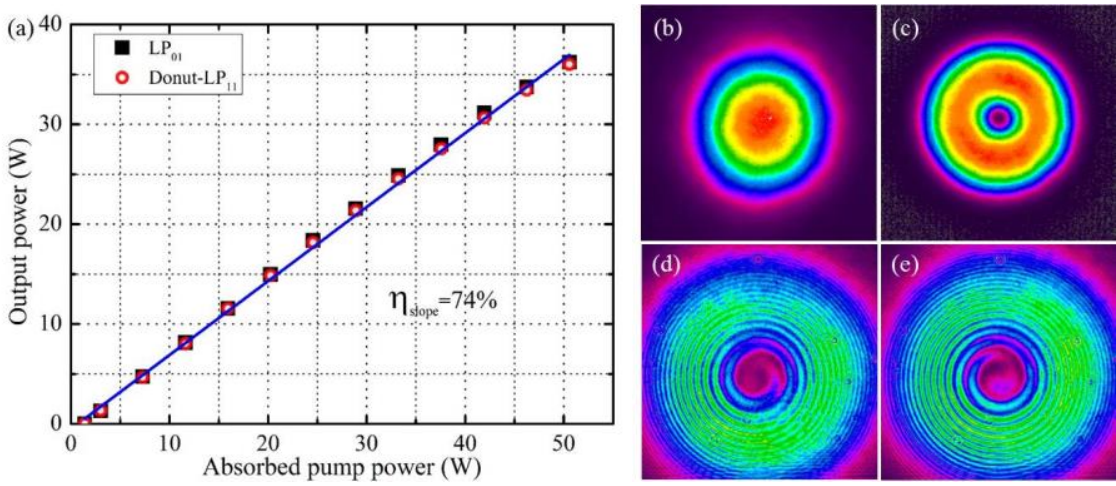

Fig. 2 (a) Output power for $\mathrm{LP}_{01}$ and donut $\mathrm{LP}_{11}$ modes as a function of absorbed pump power; Experimental far-field intensity distributions at the maximum output power: (b) the $\mathrm{LP}_{01}$ mode, (c) donut-shaped $\mathrm{LP}_{11}$ mode; (d)-(e) interference patterns of donut-shaped $\mathrm{LP}_{11}$ modes after superposition with a reference beam with a spherical wavefront.

linear polarizations with different orientations. The polarization extinction ratio (PER) was measured to be $\sim 8.7 \mathrm{~dB}$ for donut-shaped $\mathrm{LP}_{11}$ mode and $\sim 10.5 \mathrm{~dB}$ for the fundamental $\mathrm{LP}_{01}$ mode. The phase-front shape of the donut $\mathrm{LP}_{11}$ mode was measured using Mach-Zehnder interferometer. The resultant spiral fringe pattern in clockwise or anticlockwise shown in Fig. 2 (d)-(e) verified that the excited donut $\mathrm{LP}_{11}$ mode had a phase singularity at the center of beam. The handedness of the vortex beam could be switched in a repeatable manner by slightly adjusting the angle of the plane high reflectivity mirror in the external feedback cavity.

\section{Conclusion}

In summary, we have demonstrated a simple technique for selecting different transverse modes from a few-mode $\mathrm{Yb}$-doped fiber laser using an intracavity polarization filter exploiting the difference in polarization behavior for each mode. A maximum power of $36 \mathrm{~W}$ for both $\mathrm{LP}_{01}$ and donut $\mathrm{LP}_{11}$ modes with helical phase front was obtained with a slope efficiency of $74 \%$. To the best of our knowledge, this is the first demonstration of direct generation of optical vortices from a fiber laser oscillator. The prospects for further improvement in performance will be discussed.

\section{References}

[1]. P. Z. Dashti, F. Alhassen, and H. P. Lee, "Observation of orbital angular momentum transfer between acoustic and optical vortices in optical fiber," Physical Review Letters 96 (2006).

[2]. D. McGloin, N. B. Simpson, and M. J. Padgett, "Transfer of orbital angular momentum from a stressed fiber-optic waveguide to a light beam," Applied Optics 37, 469-472 (1998).

[3]. Y. Tanaka, "High power picosecond vortex laser based on a large-mode-area fiber amplifier," Optics Express 17, 14362-14366 (2009).

[4]. N. Andermahr, and C. Fallnich, "Interaction of transverse modes in a single-frequency few-mode fiber amplifier caused by local gain saturation," Optics Express 16, 8678-8684 (2008).

[5]. N. Andermahr, T. Theeg, and C. Fallnich, "Novel approach for polarization-sensitive measurements of transverse modes in few-mode optical fibers," Appl Phys B-Lasers O 91, 353-357 (2008). 